\title{
IMPACTO NA SAÚDE HUMANA DO MATERIAL PARTICULADO ORIUNDO DA POLUIÇÃO ATMOSFÉRICA
}

\author{
Clara Rodrigues Pereira1; Lílian Lefol Nani Guarieiro²
}

${ }^{1}$ Centro Universitário Senai Cimatec; Av. Orlando Gomes, 1845, Piatã; Salvador/BA; clara.r.pereira@gmail.com

${ }^{2}$ Centro Universitário Senai Cimatec; Av. Orlando Gomes, 1845, Piatã; Salvador/BA

Resumo: Estudos mostram que a exposição ao material particulado (MP) oriundo de processos de combustão veicular pode causar sérios riscos à saúde humana, uma vez que o grau de impacto do MP no sistema respiratório irá depender do seu tamanho e composição. Assim, o objetivo deste trabalho foi realizar uma revisão sistemática em busca de informações que podem abordar a problemática em questão, evidenciando causas e soluções que possam permitir um maior entendimento do tema. Foi possível concluir que a China é um país que se destaca no número de mortes causadas pela emissão de MP, diante de um cenário urbano altamente poluído. Além disso, partículas finas (MP2,5) são as mais recorrentes de incidência de doenças respiratórias, uma vez que são emitidas principalmente por atividades industriais e emissões veiculares.

Palavras-Chave: material particulado; saúde humana; sistema respiratório; emissões veiculares.

\section{IMPACT ON HUMAN HEALTH OF PARTICULAR MATERIAL ARISING FROM ATMOSPHERIC POLLUTION}

\begin{abstract}
Studies show that exposure to particulate matter (PM) from vehicular combustion processes can pose serious risks to human health since the degree of impact of PM on the respiratory system will depend on its size and composition. Thus, the objective of this work was to perform a systematic review in search of information that can address the problem in question, highlighting causes and solutions that may allow a greater understanding of the topic. It can be concluded that China is a country that stands out in the number of deaths caused by the emission of PM, given a highly polluted urban scenario. In addition, fine particles (MP2.5) are the most recurrent incidence of respiratory disease, as they are emitted mainly by industrial activities and vehicular emissions.
\end{abstract}

Keywords: particulate matter; human health; respiratory system; vehicle emissions. 


\section{INTRODUÇÃO}

A evolução global das cidades urbanas no que tange o desenvolvimento da tecnologia e o aprimoramento das atividades industriais contribui consideravelmente para a poluição do ar. A problemática chama atenção de diversos órgãos que buscam cada vez mais por ações que possam reduzir a poluição atmosférica, a exemplo da Organização das Nações Unidas que apresentou os 17 objetivos para melhorar o mundo, em um delta de tempo para cumpri-los até o ano de 2030. A sua principal missão é determinar ações que possam acabar com a pobreza, promover a prosperidade e o bem-estar para todos, proteger o meio ambiente e enfrentar as mudanças climáticas [1]. Dentro deste panorama, o objetivo 7 (Energia limpa e acessível) se destaca, uma vez que abrange medidas afim reduzir as principais fontes de poluição no ambiente externo, como a participação de energia renovável na matriz energética global e o acesso a tecnologias com energia limpa [1].

Diante deste cenário, substâncias que são emitidas por indústrias, escapamento de automóveis e outras atividades urbanas são depositadas nos pavimentos de rodovias e nos espaços de circulação humana, contribuindo para o aumento da poluição do ar e à exposição do indivíduo a este ambiente [2]. Dados da World Health Globalization (WHO) do ano de 2016 mostraram que a poluição do ar, em cidades e áreas rurais, é responsável por 4,2 milhões de mortes prematuras por ano, devido à exposição ao material particulado (MP), que são causadores de doenças cardiovasculares, respiratórias e cancerígenas [3]. Estas informações mostram o nível de impacto que o MP pode ocasionar na saúde humana, mas por quê? O que faz como que estas partículas apresentem danos ao nosso corpo? Como elas são formadas, qual a sua origem?

O MP atmosférico constitui de misturas de partículas sólidas suspensas no ar que variam em tamanho e composição química. Podem ser compostos por nitratos, sulfatos, carbono orgânico e elementar, compostos orgânicos, compostos biológicos e metais (ferro, cobre, níquel, zinco e vanádio) [4]. Eles são originados de fontes naturais, as quais não envolvem atividades humanas (partículas de sal marinho, areia soprada pelo vento, cinzas vulcânicas) e fontes antropogênicas, que são oriundas de processos urbanos (processos industriais, veículos e combustíveis) [5].

O comprimento do diâmetro aerodinâmico do MP é o principal fator que determina a capacidade de transporte da partícula na atmosfera e a capacidade de penetração no sistema respiratório [4]. O MP pode ser classificado por três grupos: Partículas grossas $\mathrm{MP}_{10}$ (entre 2,5 e $10 \mu \mathrm{m}$ de diâmetro), partículas finas $\mathrm{MP}_{2,5}$ (diâmetro menor que $2,5 \mu \mathrm{m}$ ) e partículas ultrafinas $\mathrm{MP}_{1}$ (diâmetro menor que $0,1 \mu \mathrm{m}$ ) [4]. As partículas grossas ( $\mathrm{MP}_{10}$ ) são definidas como partículas primárias, por serem diretamente emitidas para atmosfera e as partículas finas $\left(\mathrm{MP}_{2,5}\right)$ são classificadas como secundárias uma vez que a sua formação na atmosfera ocorre através de reações químicas [4].

A emissão de material particulado apresenta um papel de destaque na literatura, ao evidenciar sérias doenças que podem ser causadas pelo impacto do MP no trato respiratório, como doenças cardiovasculares, doenças respiratórias e doenças do sistema nervoso [2]. Desta forma, alguns meios de monitoramento já vêm sendo adotados para identificar o nível de MP emitido pelas atividades urbanas, a 
exemplo de estações fixas ou móveis que possuem ferramentas adequadas para definir quais os tamanhos que são mais emitidos e a sua fonte de emissão [3]. Contudo, além do monitoramento, é de suma importância a avaliação do impacto da exposição de seres humanos ao MP. Assim, modelagem matemática que vise a simulação da deposição do MP no pulmão é uma das ferramentas importante na avaliação do impacto deste na saúde [6].

Neste contexto, o objetivo deste estudo foi realizar uma revisão sistemática sobre o impacto do material particulado na saúde humana quando emitido pelos processos de urbanização.

\section{METODOLOGIA}

A metodologia aplicada para o desenvolvimento deste estudo foi a revisão sistemática (Figura 1). Este método foi desenvolvido conforme as seguintes etapas: i) as palavras-chaves utilizadas em inglês foram "particulate matter", "dry deposition", "wet deposition", "vehicular emission", "lung depositiont"; ii) as bases de dados utilizadas para pesquisa foram Scopus (www.scopus.com), science direct (www.sciencedirect.com) e 0 capes (www-periodicos-capes-govbr.ez68.periodicos.capes.gov.br); iii) o período de busca estabelecido foi de 2009 a 2019; iv) foi verificado se o título dos artigos possuía a palavra - chave determinada; v) foi realizada a leitura dos resumos, conforme o filtro estabelecido (Figura 1).

Figura 1. Fluxograma da metodologia de Revisão sistemática.

Revisão Sistemática

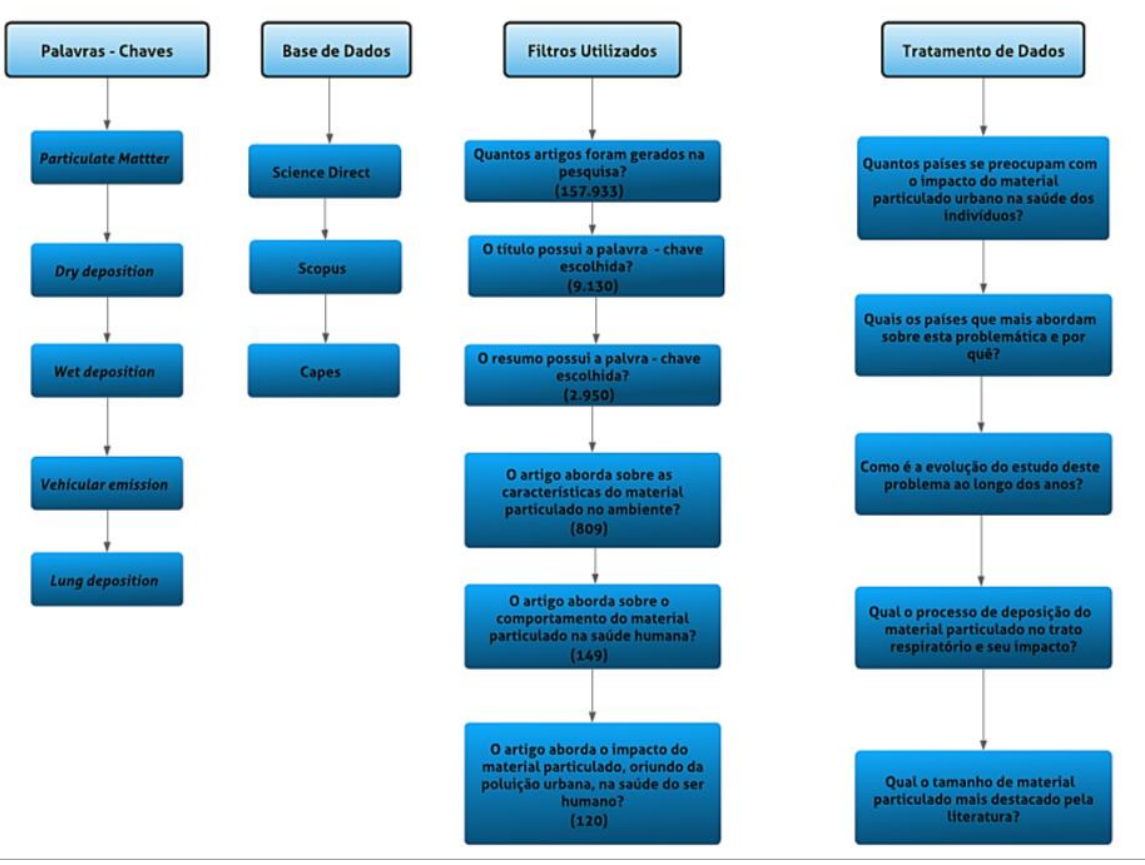




\section{RESULTADOS E DISCUSSÃO}

Uma das etapas utilizadas na revisão sistemática, foi o intervalo de tempo (2009 a 2019) estipulado na busca dos materiais referentes à temática apresentada. Assim, a Figura 2 representa como o estudo em questão evoluiu ao passar dos anos, comprovando que o tema abordado tem despertado interesse, apresentando um elevado grau de importância (Total de 120 artigos).

Figura 2. Relação percentual de artigos publicados sobre o tema ao longo dos anos.

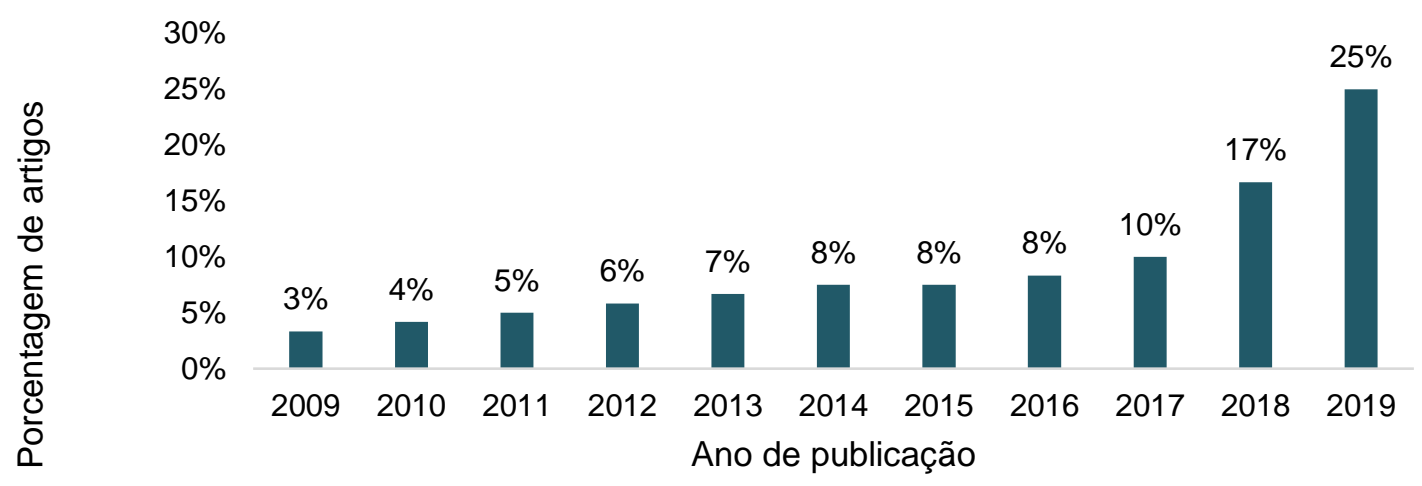

A partir da revisão sistemática realizada neste trabalho foi possível direcionar a pesquisa ao impacto que o MP, oriundo de processos de urbanização, pode causar na saúde humana. Foram obtidos 120 artigos pertinentes ao tema. Ao selecionar os trabalhos, foram identificados os países que mais pesquisam sobre essa problemática (Figura 3).

Figura 3. Relação dos países que mais publicam sobre o tema da pesquisa.
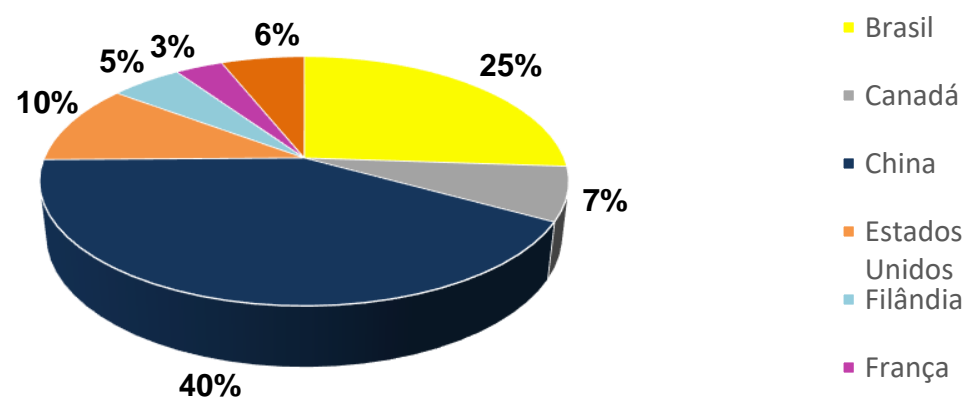

A China apresentou $40 \%$ dos artigos selecionados para o estudo, que destacam o impacto do MP na saúde dos indivíduos, gerado nas cidades urbanas (Figura 3). Isso se deve ao fato de que a poluição atmosférica na China, tornou-se o quarto principal risco à saúde, apresentando o MP como o principal poluente atmosférico [7].

O grau elevado de poluição na China é recorrente da rápida urbanização e desenvolvimento econômico, onde a maioria dos MP é emitida por atividades 
antropogênicas (atividades industriais, emissões de veículos) [7]. Desta forma, a exposição a este particulado representa uma vulnerabilidade na saúde humana, quando este é depositado no trato respiratório, devido aos altos níveis de metais e hidrocarbonetos policíclicos aromáticos presentes no MP [7].

A Figura 4 ratifica esta afirmação ao mostrar por meio de um mapa ilustrativo, dados de 2016 que apresentam em cada país, os maiores índices de mortalidade devido à inalação de MP no pulmão. No mapa, a China apresenta-se com a cor correspondente a um índice de mortalidade entre 1500 e 1999 milhões, tendo como um valor exato de 1.830 milhões de mortes causadas pela emissão de MP [3].

Figura 4. Mortalidade e carga de doenças causadas pela poluição do ar ambiente.

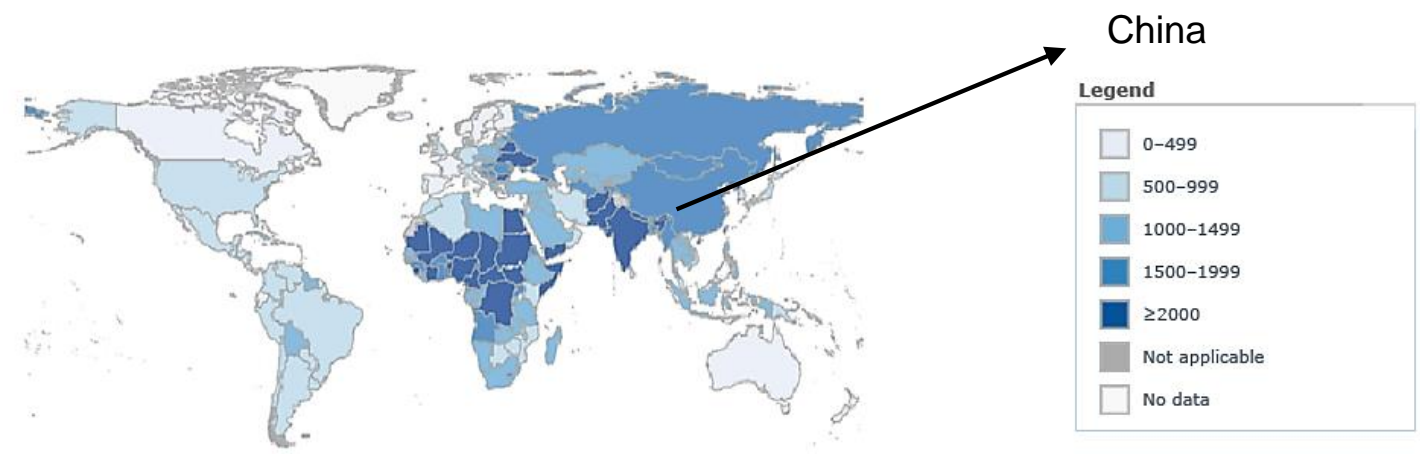

Fonte: WHO, 2018 [8].

A exposição ao MP é muito influenciada pelas condições locais, como clima, estações do ano, topografia, fontes de partículas, dentre outros. Porém, a inalação é o meio mais importante para o contato do organismo humano com o MP, de forma que o seu grau de impacto está correlacionado com o tamanho da partícula depositada no aparelho respiratório [9]. A deposição do MP no pulmão ocorre de forma que quanto menor a partícula, maior a capacidade de absorver compostos orgânicos e inorgânicos e maior a capacidade de penetrar no trato respiratório [4]. As partículas grossas $\left(\mathrm{MP}_{10}\right)$ ao serem inaladas são alojadas na traqueia (parte superior da garganta), devido ao atrito do $\mathrm{MP}_{10} \mathrm{com}$ os pelos nasais, assim são mais difíceis de penetrar no aparelho expiratório [4].

As partículas finas $\left(\mathrm{MP}_{2,5}\right)$ apresentaram um destaque na literatura como provedoras de doenças de alto risco e pelo maior grau de emissão ser oriundo de escapamento de veículos, produto da combustão incompleta dos motores de combustão interna [10]. $\mathrm{O} \mathrm{MP}_{2,5}$ ao ser inalado percorrem os bronquíolos e alvéolos (onde ocorre a troca gasosa), podendo penetrar na corrente sanguínea ocasionando problemas de saúde significativos [4]. O grau de impacto do $\mathrm{MP}_{10}$ e $\mathrm{MP}_{2,5}$ é muito encontrados na literatura ao serem relacionados com o de outros tamanhos de partículas, como o $\mathrm{MP}_{1,0}$, que assim como o $\mathrm{MP}_{2,5}$ apresenta uma capacidade ainda maior de penetrar mais profundamente no ser humano e agravar a asma, bronquite e outros problemas respiratórios, levando a sintomas cardiovasculares [11].

A exposição ao MP pode promover a incidência de doenças de alto risco como: problemas respiratórios, exacerbação de respiração crônica, diminuição da função 
pulmonar, mortalidade prematura, além dos riscos carcinogênicos e mutagênicos [4]. O MP emitido pela queima de combustíveis é composto por carbono, dióxido de enxofre, hidrocarbonetos não queimados e alguns metais, de forma que podem ter uma forte relação com o risco de câncer de pulmão, por ocasionarem danos ao DNA humano, ou seja, quebras e alterações na cadeia genética (efeitos mutagênicos) [12].

Assim, por apresentarem em sua composição compostos genotóxicos (mutagênicos), as partículas emitidas, principalmente as de tamanhos menores, possuem uma alta capacidade de induzir efeitos carcinogênicos que são capazes de desenvolver sintomas de câncer pulmonar, além de outros efeitos como a falta de ar, desconforto e dor no peito, tosse forte e chiado [4]. Crianças estão mais sujeitas a serem afetadas pelo impacto do MP do trato respiratório uma vez que pode comprometer o desenvolvimento e função pulmonar. Desta forma, uma exposição em um nível excessivo de partículas finas pode aumentar consideravelmente os problemas respiratórios e os efeitos carcinogênicos [4].

\section{CONCLUSÃO}

Este artigo teve como foco a abordagem do impacto que o MP pode ocasionar na saúde humana, utilizando a revisão sistemática como recurso para obter na literatura informações que comprovem a problemática citada. Como um dos resultados desta metodologia, foi notável, a evolução da busca por estudos relacionados a esta temática ao longo dos anos. A China foi identificada como o país que mais publica artigos sobre o tema e isso se justifica pelo fato dela apresentar 0 maior grau de poluição decorrente de atividades antropogênicas. A intensa ocorrência deste tipo de atividade proporciona uma alta emissão de MP finos, que ao serem depositados no trato respiratório podem causar danos irreversíveis à saúde humana.

\section{REFERÊNCIAS}

1 ONU. 17 Objetivos para transformar nosso mundo. Disponível em: <https://nacoesunidas.org/pos2015/ods7/>. Acesso em: 02 ago. 2019.

${ }^{2} \mathrm{ALI}$, Muhammad Ubaid et al. A systematic review on global pollution status of particulate matter-associated potential toxic elements and health perspectives in urban environment. Environmental geochemistry and health, p. 1-32, 2018.

3 WHO. Ambient (outdoor) air quality and health. Disponível em: <https://www.who.int/en/news-room/fact-sheets/detail/ambient-(outdoor)-air-qualityand-health/>. Acesso em: 05 ago. 2019

${ }^{4} \mathrm{KIM}$, Ki-Hyun; KABIR, Ehsanul; KABIR, Shamin. A review on the human health impact of airborne particulate matter. Environment international, v. 74, p. 136-143, 2015. 
${ }^{5} \mathrm{HU}$, Zimei et al. Concentrations and source apportionment of particulate matter in different functional areas of Shanghai, China. Atmospheric Pollution Research, v. 5, n. 1, p. 138-144, 2014.

6 DĖDELE், Audrius; MIŠKINYTĖ, Auksè. Seasonal and site-specific variation in particulate matter pollution in Lithuania. Atmospheric Pollution Research, v. 10, n. 3, p. 768-775, 2019.

7 WANG, Shenbo et al. Size-fractionated particulate elements in an inland city of China: Deposition flux in human respiratory, health risks, source apportionment, and dry deposition. Environmental pollution, v. 247, p. 515-523, 2019.

${ }^{8} \mathrm{WHO}$. Mortality and burden of disease from ambient air pollution. Disponível em: <https://www.who.int/en/news-room/fact-sheets/detail/ambient-(outdoor)-airquality-and-health/>. Acesso em: 05 ago. 2019.

${ }^{9}$ GUARIEIRO, L. L. N.; GUARIEIRO, A. L. N. Vehicle Emissions: What Will Change with Use of Biofuel? In: Biofuels - Economy, Environment and Sustainability. [s.I.] InTech, 2013.

${ }^{10}$ WONG, Yee Ka et al. Estimating contributions of vehicular emissions to PM2. 5 in a roadside environment: A multiple approach study. Science of The Total Environment, v. 672, p. 776-788, 2019.

${ }^{11} \mathrm{KARRI}$, Rama Rao et al. Modeling airborne indoor and outdoor particulate matter using genetic programming. Sustainable Cities and Society, v. 43, p. 395-405, 2018.

${ }^{12}$ GUERRERA, Elena et al. Cytotoxicity and genotoxicity of size-fractionated particulate matter collected in underground workplaces. Air Quality, Atmosphere \& Health, v. 12, n. 3, p. 359-367, 2019. 